TAIWANESE JOURNAL OF MATHEMATICS

Vol. 5, No. 3, pp. 633-640, September 2001

This paper is available online at http://www.math.nthu.edu.tw/tjm/

\title{
REMARKS ON POINCARÉ INEQUALITY AND ITS APPLICATIONS TO ELLIPTIC BOUNDARY VALUE PROBLEMS
}

\author{
Shyuh-yaur Tzeng ${ }^{\dagger}$
}

\begin{abstract}
We study Poincaré inequality on unbounded domains and its applications to the semilinear elliptic equation $-\triangle u=u^{p}$.
\end{abstract}

\section{INTRODUCTION}

In the study of elliptic boundary value problems, the Poincare inequality has frequently been used to obtain various estimates for the solutions. Such kinds of inequalities are usually more difficult to obtain for unbounded domains. Our aim in this note is to investigate under what conditions the Poincaré inequality holds, that is, there is a constat $C=C(\Omega)$ such that

$$
\int_{\Omega}|u(x)|^{2} d x \leq C \int_{\Omega}|\nabla u(x)|^{2} d x
$$

for all $u \in H_{0}^{1}(\Omega)$, where $\Omega$ is an open subset of $\mathbb{R}^{N}$.

The detailed analysis is given in the next section. Some applications to elliptic boundary value problems will be discussed in Section 3 .

\section{Poincaré Inequality}

Definition 1. An open subset of $\mathbb{R}^{N}$ is called a P-domain if (1) holds. For any open subset of $\mathbb{R}^{N}$, define

$$
C(\Omega)=\sup _{u \in H_{0}^{1}(\Omega), u \neq 0} \frac{\int_{\Omega}|u(x)|^{2} d x}{\int_{\Omega}|\nabla u(x)|^{2} d x} .
$$

Received June 7, 2000; revised September 30, 2000.

Communicated by C.-S. Lin.

2000 Mathematics Subject Classification: 35J20, 35K25.

Key words and phrases: Poincare inequality, semilinear elliptic equation, unbounded domain.

${ }^{\dagger}$ Research was partially supported by the National Science Council of Republic of China. 
It is known that $\mathbb{R}^{N}$ is not a P-domain. On the other hand, any bounded open set in $\mathbb{R}^{N}$ is a P-domain. For unbounded domains, it has been shown [9] that $\left\{\left(x_{1}, x_{N}^{\prime}\right) \in \mathbb{R}^{N}: x_{1} \in \mathbb{R},\left|x_{N}^{\prime}\right|<1\right\}$ is a P-domain. Nevertheless, for a general unbounded set, it is no easy matter to verify if such a set is a P-domain. The first result of this paper is to give a criterion for an unbounded set to be a P-domain.

Theorem 1. Let $\Omega_{1}$ and $\Omega_{2}$ be two P-domains. If $\Omega_{1} \cap \Omega_{2}$ is bounded, then $\Omega=\Omega_{1} \cup \Omega_{2}$ is a P-domain.

Proof. Since $\Omega_{1} \cap \Omega_{2}$ is bounded, $\Omega_{1} \cap \Omega_{2}$ is contained in an open ball $B$ of radius $r$ with center at 0 . Consider a smooth function $\phi$ which satisfies

$$
\phi(t)= \begin{cases}1, & |x| \leq r \\ 0, & |x| \geq 2 r\end{cases}
$$

Let $C_{0}=C(B), C_{1}=C\left(\Omega_{1}\right)$ and $C_{2}=C\left(\Omega_{2}\right)$. A straightforward calculation yields

$$
\int_{\Omega} u^{2} d x \leq 2\left(\int_{\Omega}(\phi u)^{2} d x+\int_{\Omega}[(1-\phi) u]^{2} d x\right)
$$

and

$$
\begin{aligned}
& \int_{\Omega}(\phi u)^{2} d x=\int_{B}(\phi u)^{2} d x \leq C_{0} \int_{B}|\nabla(\phi u)|^{2} d x \\
& \leq 5 C_{0} \int_{B}|\nabla u|^{2} d x+\frac{8 C_{0}}{r} \int_{B} u^{2} \leq 5 C_{0} \int_{\Omega}|\nabla u|^{2} d x+\frac{8 C_{0}}{r} \int_{\Omega} u^{2} .
\end{aligned}
$$

Similarly, we obtain

$$
\begin{aligned}
& \int_{\Omega}[(1-\phi) u]^{2} d x=\int_{\Omega_{1}}[(1-\phi) u]^{2} d x+\int_{\Omega_{2}}[(1-\phi) u]^{2} d x \\
& \leq C\left(\Omega_{1}\right) \int_{\Omega_{1}}|\nabla[(1-\phi) u]|^{2} d x+C\left(\Omega_{2}\right) \int_{\Omega_{2}}|\nabla[(1-\phi) u]|^{2} d x \\
& \leq 5\left(C_{1}+C_{2}\right) \int_{\Omega}|\nabla u|^{2} d x+\frac{8\left(C_{1}+C_{2}\right)}{r} \int_{\Omega} u^{2} .
\end{aligned}
$$

Putting (3)-(5) together yields

$$
\int_{\Omega} u^{2} d x \leq 5\left(C_{0}+C_{1}+C_{2}\right) \int_{\Omega}|\nabla u|^{2} d x+\frac{8\left(C_{0}+C_{1}+C_{2}\right)}{r} \int_{\Omega} u^{2} .
$$

If $r$ is large enough, then

$$
\int_{\Omega} u^{2} d x \leq 5\left(C_{0}+C_{1}+C_{2}\right) \int_{\Omega}|\nabla u|^{2} d x
$$


which completes the proof.

Next, we give a criterion for sets which are not P-domains.

Definition 2. An open subset $\Omega$ in $\mathbb{R}^{N}$ is called a $B$-domain if for any $r>0$, there exists an open ball of radius $r$ contained in $\Omega$.

Theorem 2. Any B-domain is not a P-domain.

In the proof of Theorem 2, we will use the following lemma.

Lemma 1. Let $B_{r}$ be an open ball of radius $r$. Then $C\left(B_{r}\right) \rightarrow \infty$ as $r \rightarrow \infty$.

Proof. By change of variables, we get $C\left(B_{r}\right)=r^{2} C\left(B_{1}\right)$, from which the result follows.

Proof of Theorem 2. For any $r>0, \Omega$ contains an open ball $B_{r}$. Since $H_{0}^{1}\left(B_{r}\right) \subseteq H_{0}^{1}(\Omega)$, it follows from Lemma 1 that $C(\Omega)=\infty$. Thus the proof is complete.

Let $B_{r}(0)$ be the open ball of radius $r$ with center at 0 . As more concrete examples of P-domains, we have

Example 1. Let

$$
D_{1}=\left\{\left(x_{1}, x_{N}^{\prime}\right) \in \mathbb{R}^{N}:\left|x_{N}^{\prime}\right|<1, x_{1}>0\right\} .
$$

Then by Theorem $1, D_{1} \cup B_{r}(0)$ is a P-domain.

Example 2. Let

$$
D_{2}=\left\{\left(x_{1}, \cdots, x_{N}\right) \in \mathbb{R}^{N}: x_{1}^{2}+\cdots+x_{N-1}^{2}<1, x_{N}>0\right\} .
$$

Then $D_{1} \cup D_{2}$ is a P-domain.

\section{Applications to Elliptic Boundary Value Problems}

As an application of our previous results, we now study the existence of positive solutions of

$$
\begin{array}{r}
-\triangle u=u^{p}, u>0 \text { in } \Omega, \\
u \in H_{0}^{1}(\Omega),
\end{array}
$$


where $p>1$ if $N=1,2$ and $p \in(1,(N+2) /(N-2))$ if $N>2$. To seek solutions of $(E)$, we define

$$
J(u)=\frac{1}{2} \int_{\Omega}|\nabla u|^{2} d x-\frac{1}{p+1} \int_{\Omega}|u|^{p+1} d x
$$

and look for the minimizers of $J$ on the manifold:

$$
M=M(\Omega)=\left\{u \in H_{0}^{1}(\Omega) \backslash\{0\}: J^{\prime}(u) u=0\right\} .
$$

Set

$$
\alpha=\alpha(\Omega)=\inf _{u \in M} J(u) .
$$

It has been shown $[2,3,14]$ that if $u$ is a minimizer of $J$ on $M$, then $|u|$ is a positive solution of (E). We are going to investigate under what conditions there is a minimizer of $J$ on $M$.

Definition 3. A P-domain $\Omega$ is periodic if there exist a partition $\left\{Q_{m}\right\}$ and a set $\left\{y_{m}\right\}$ in $\mathbb{R}^{N}$ which satisfy the following conditions:

(a) $\left\{y_{m}\right\}$ forms a subgroup of $\mathbb{R}^{N}$,

(b) $Q_{0}$ is bounded,

(c) $Q_{m}=Q_{0}+y_{m}$, and

(d) there exists a constant $C=C\left(\Omega, Q_{m}\right)$ such that

$$
\int_{Q_{m}}|u(x)|^{2} d x \leq C \int_{Q_{m}}|\nabla u(x)|^{2} d x
$$

for all $u \in H_{0}^{1}(\Omega)$ and $m \in \mathbb{Z}$.

Theorem 3. If $\Omega$ is a periodic P-domain, then $J$ has a minimizer on $M$.

Proof. Let $\left\{u_{n}\right\} \subset M(\Omega)$ be a sequence which satisfies

$$
\lim _{n \rightarrow \infty} J\left(u_{n}\right)=\alpha(\Omega) .
$$

Let

$$
d_{n}=\max _{m}\left(\int_{Q_{m}}\left|u_{n}\right|^{p+1} d x\right)^{1 /(p+1)}
$$


Then

$$
\begin{aligned}
\alpha(\Omega)+o(1) & =J\left(u_{n}\right)=\left(\frac{1}{2}-\frac{1}{p+1}\right) \int_{\Omega}\left|u_{n}\right|^{p+1} d x \\
& =\left(\frac{1}{2}-\frac{1}{p+1}\right) \sum_{m} \int_{Q_{m}}\left|u_{n}\right|^{p+1} d x \\
& \leq d_{n}^{p-1}\left(\frac{1}{2}-\frac{1}{p+1}\right) \sum_{m}\left(\int_{Q_{m}}\left|u_{n}\right|^{p+1} d x\right)^{\frac{2}{p+1}} \\
& \leq \operatorname{cd}_{n}^{p-1}\left(\frac{1}{2}-\frac{1}{p+1}\right) \sum_{m} \int_{Q_{m}}\left|\nabla u_{n}\right|^{2} d x \\
& =\operatorname{cd}_{n}^{p-1}\left(\frac{1}{2}-\frac{1}{p+1}\right) \int_{\Omega}\left|\nabla u_{n}\right|^{2} d x=\operatorname{cd}_{n}^{p-1} \alpha(\Omega)+o(1)
\end{aligned}
$$

for some positive constant $c$ which is independent of $m$. Therefore, there is a $\delta>0$ such that $d_{n}>\delta$ for all $n$.

Next, we claim that there exists a $u \in M(\Omega)$ such that

$$
J^{\prime}(u)=0 \text { and } J(u)=\alpha(\Omega) .
$$

For each $n$, find a $Q_{n}$ such that

$$
\int_{Q_{n}}\left|u_{n}\right|^{p+1} d x>\frac{\delta}{2} .
$$

Let $\nu_{n}(x)=u_{n}\left(x+y_{n}\right)$. It follows from (8) that

$$
\lim _{n \rightarrow \infty} J\left(\nu_{n}\right)=\alpha(\Omega) .
$$

Using the standard deformation theory $[2,8]$ yields

$$
J^{\prime}\left(\nu_{n}\right) \rightarrow 0 \text { as } n \rightarrow \infty .
$$

Along a subsequence, $\nu_{n} \rightarrow u$ weakly in $H_{0}^{1}(\Omega)$. Then using (12) and Sobolev compact imbedding theorem yields

$$
\int_{Q_{0}}|u|^{p+1} d x \geq \frac{\delta}{2}
$$

Consequently, $u \not \equiv 0$. Moreover, by (14),

$$
J^{\prime}(u)=0,
$$


which shows $u \in M(\Omega)$. Now (13) together with Fatou's Lemma gives

$$
\begin{aligned}
\alpha(\Omega) & \leq J(u)=\left(\frac{1}{2}-\frac{1}{p+1}\right) \int_{\Omega}|u|^{p+1} d x \\
& \leq \liminf \left(\frac{1}{2}-\frac{1}{p+1}\right) \int_{\Omega}\left|\nu_{n}\right|^{p+1} d x=\alpha(\Omega),
\end{aligned}
$$

which completes the proof.

Example 3. The following are some simple examples of periodic P-domains.

$D_{3}=O \times \mathbb{R}^{m}$, where $O$ is a bounded domain in $\mathbb{R}^{p}, m \geq 1, p \geq 1$,

$D_{4}=\left\{(x, y) \in \mathbb{R}^{2}: \sin x<y<1+\sin x\right\}$,

$D_{5}=\left\{(x, y, z) \in \mathbb{R}^{3}: x=(\cos \theta)(2+\sin z), y=(\sin \theta)(2+\sin z), z \in R, \theta \in \mathbb{R}\right\}$.

Remark 1. As proved in [10], the only solution of (E) is $u \equiv 0$ if $\Omega=D_{1}$, where $D_{1}$ was defined in Example 1. Thus we cannot expect that (E) has positive solutions on all P-domains.

Theorem 4. Let $\Omega_{1}$ and $\Omega_{2}$ be two P-domains such that $\Omega_{1} \cap \Omega_{2}$ is bounded. If $\alpha\left(\Omega_{1}\right) \leq \alpha\left(\Omega_{2}\right)$ and $J$ has minimizers on $M\left(\Omega_{1}\right)$, then there is a positive solution on $\Omega$.

Proof. Let $\alpha_{1}=\alpha\left(\Omega_{1}\right)$ and $\alpha_{2}=\alpha\left(\Omega_{2}\right)$. It is not difficult to show, by using the Maximum Principle, that

$$
\alpha(\Omega)<\alpha_{1}
$$

Let $\left\{u_{n}\right\} \subset M(\Omega)$ be a sequence which satisfies

$$
J\left(u_{n}\right) \rightarrow \alpha(\Omega) \text { as } n \rightarrow \infty .
$$

Since $\Omega_{1} \cap \Omega_{2}$ is bounded, there is an $r>0$ such that $\Omega_{1} \cap \Omega_{2} \subset B_{r}(0)$.

We now claim there is a $d>0$ such that

$$
\int_{|x| \leq 2 r} u_{n}^{2} d x \geq d
$$

for all $n$. For otherwise, there exists a subsequence $\left\{u_{n}\right\}$ such that

$$
\lim _{n \rightarrow \infty} \int_{|x| \leq 2 r} u_{n}^{2} d x=0 .
$$


Let $\Omega_{1}^{\prime}=\Omega_{1} \backslash \overline{B_{r}(0)}$ and $\Omega_{2}^{\prime}=\Omega_{2} \backslash \overline{B_{r}(0)}$. Then by (21), there exist $\left\{u_{n, 1}\right\} \subset$ $H_{0}^{1}\left(\Omega_{1}^{\prime}\right)$ and $\left\{u_{n, 2}\right\} \subset H_{0}^{1}\left(\Omega_{2}^{\prime}\right)$ such that

$$
\lim _{n \rightarrow \infty}\left\|u_{n}-u_{n, 1}-u_{n, 2}\right\|=0
$$

and

$$
u_{n, 1}+u_{n, 2} \in M(\Omega)
$$

for all $n$. Consequently, $\alpha(\Omega) \geq \min \left(\alpha_{1}, \alpha_{2}\right)$, which contradicts (18). The rest of the proof is essentially the same as that of (11).

The proof of Theorem 4 also yields

Corollary 1. If $\Omega_{1}$ and $\Omega_{2}$ are two periodic P-domains and $\Omega_{1} \cap \Omega_{2}$ is bounded, then there is a positive solution on $\Omega_{1} \cup \Omega_{2}$.

A consequence of Theorem 4 is the following:

Example 4. Let $\Omega=D_{1} \cup B_{r}(0)$. If $r$ is large enough, then $J$ has a minimizer on $M(\Omega)$.

Proof. It suffices to show that

$$
\lim _{r \rightarrow \infty} \alpha\left(B_{r}\right)=0 .
$$

For any $r>1$, take $u_{r} \in M\left(B_{r}\right)$ such that

$$
\sup _{r>1} \int_{B_{r}} u_{r}^{2} d x<\infty
$$

and

$$
C\left(B_{r}\right)<\frac{2 \int_{B_{r}}\left|u_{r}(x)\right|^{2} d x}{\int_{B_{r}}\left|\nabla u_{r}(x)\right|^{2} d x} .
$$

Applying the Hölder inequality and the Sobolev inequality yields

$$
\begin{aligned}
\alpha\left(B_{r}\right) & \leq \frac{1}{2} \int_{B_{r}}\left|\nabla u_{r}\right|^{2} d x-\frac{1}{p+1} \int_{B_{r}}\left|u_{r}\right|^{p+1} d x \\
& =\left(\frac{1}{2}-\frac{1}{p+1}\right) \int_{B_{r}}\left|\nabla u_{r}\right|^{2} d x \leq \frac{c}{C\left(B_{r}\right)}
\end{aligned}
$$

for some constant $c$. Then using Lemma 1 yields

$$
\lim _{r \rightarrow \infty} \alpha\left(B_{r}\right)=0
$$




\section{REFERENCES}

1. R. A. Adams, Sobolev Spaces, Academic Press, New York, 1975.

2. A. Ambrosetti and P. H. Rabinowitz, Dual variational methods in critical point theory and applications, J. Funct. Anal 14 (1973), 394-381.

3. K.-C. Chang, Infinite Dimensional Morse Theory and Multiple Solution Problems, Birkhäuser, Boston, 1993.

4. C.-C. Chen and C. -S. Lin, Uniqueness of ground state solutions of $\triangle u+f(u)=0$ in $\mathbb{R}^{N}, N \geq 3$, Comm. Partial Differential Equations 16 (1991), 1549-1572.

5. K.-S. Cheng and W.-M. Ni, On the structure of the conformal scalar curvature equation on $\mathbb{R}^{n}$, Indiana Univ. Math. J. 41 (1992), 261-278.

6. W.-Y. Ding and W.-M. Ni, On the existence of a positive entire solution of a semilinear elliptic equation, Arch. Rational Mech. Anal. 91 (1986), 283-308.

7. M. del Pino and P. Felmer, Local mountain passes for seminiear elliptic problems in unbounded domains, Calc. Var. Partial Differential Equations 4 (1996), 121-137.

8. I. Ekeland, On the variational principle, J. Math. Anal. Appl. 47 (1974), 324-353.

9. M. J. Esterban, Nonlinear elliptic problems in strip-like domains: symmetry of positive vortex rings, Nonlinear Anal. 7 (1983), 365-379.

10. M. J. Esterban and P. L. Lions, Existence and non-existence results for semilinear problems in unbounded domains, Proc. Roy. Soc. Edinturgh Sect. A 93 (1982), 1-14.

11. D. Gilbarg and N. S. Trudinger, Elliptic Partial Differential Equations of Second Order, $2^{\text {nd }}$ ed., Springer-Verlag, Berlin, 1983.

12. P. L. Lions, The concentration-compactness principle in the calculus of variations, The locally compact case, Ann. Inst. H. Poincaré, Anal. Non Linéaire, 1 (1984), Part 1, 109-145; Part 2, 223-283.

13. W. C. Lien, S. Y. Tzeng and H. C. Wang, Existence of solutions of semilinear elliptic problems on unbounded domains, Differential Integral Equations 6 (1993), 12821298.

14. M. Struwe, Variational Methods, Application to Nonlinear Partial Differential Equations and Hamiltonian Systems, Springer-Verlag, Berlin, 1990.

Department of Mathematics, National Changhua University of Education Changhua, Taiwan, R.O.C. 